\title{
Sonographic soft tissue arthritic changes associated with post-stroke hemiplegic knee pain: utility of musculoskeletal ultrasound in a resource-limited setting
}

\author{
Adeniyi S. Aderibigbe ${ }^{1 A, B, C, D, E, F}$, Olushola C. Famurewa ${ }^{1 A, D, E}$, Morenikeji A. Komolafe ${ }^{2 A, D, E}$, Adeleye D. Omisore ${ }^{1 A, D, E}$, \\ Victor A. Adetiloye ${ }^{1 \mathrm{E}}$
}

'Department of Radiology, Obafemi Awolowo University, Ile-Ife, Nigeria

${ }^{2}$ Department of Internal Medicine, Obafemi Awolowo University, Ile-Ife, Nigeria

\section{Abstract}

Purpose: Post-stroke arthritic changes that may compromise rehabilitation have been described in the upper and lower limbs. We aimed at evaluating the soft tissue arthritic changes associated with pain in hemiplegic knees of stroke patients in our environment.

Material and methods: Hemiplegic and non-hemiplegic knees of 48 stroke patients as well as both knees of 26 apparently healthy controls were evaluated with a 7.5-12 MHz linear ultrasound probe. History of knee pain, and sociodemographic, laboratory, and clinical data were recorded for all study participants. Muscle tone and power as well as functional ambulatory category (FAC) were graded for the stroke patients. Data was analysed using SPSS version 20.

Results: Pain was reported more often in hemiplegic than non-hemiplegic knees ( $n=16$ vs. $n=6, p=0.021$ ). The frequencies of soft tissue arthritic changes found, which included reduced lateral and medial femoral cartilage thickness, suprapatellar effusion, and irregular cartilage-bone margin, were similar between hemiplegic and non-hemiplegic knees $(p>0.05)$. Suprapatellar effusion and reduced lateral femoral cartilage thickness were more prevalent in the hemiplegic knees compared to the healthy control knees $(p<0.05)$. Stroke patients with pes anserinus tendinosis had greater risk of hemiplegic knee pain (HKP) when compared to stroke patients without this lesion $(\mathrm{OR}=10$; 95\% CI: 1.7-61). FAC, muscle tone, and power showed no association with HKP.

Conclusions: Soft tissue arthritic changes associated with knee pain are comparable between hemiplegic and non-hemiplegic knees of stroke patients. The risk of knee pain in stroke is higher in the presence of pes anserinus tendinosis.

Key words: osteoarthritis, stroke, ultrasound, pes anserinus tendinosis, hemiplegic knee pain.

\section{Introduction}

Stroke frequently causes upper motor neuron syndrome, including weakness, spasticity, and abnormal gait pattern or synergistic movement. The impairment of lower limb function results in an asymmetric and interrupted hemiplegic gait because patients prefer to bear weight on the unaffected limb. Biomechanical changes in both limbs lead to soft-tissue injuries, and arthritic cartilage changes may be suspected $[1,2]$. When present, arthritic pathology aggravates motor dysfunction and compromises rehabilitation [3].

In hemiplegic patients, musculoskeletal problems are most prevalent in the shoulder and knee joints, with prevalence ranging from 30 to $65 \%$ in the shoulder and from 6.5 to $25.4 \%$ in the knee joint, depending on the population studied and the method of assessment used [4-6]. Also, Kong et al. [5] reported additional contralateral (non-paretic) knee pain among $2 \%$ of hemiplegic stroke patients studied. Most of the published studies comparing arthritic

Correspondence address:

Adeniyi S. Aderibigbe, Department of Radiology, Obafemi Awolowo University, Ile-Ife, Nigeria, e-mail: adeniyiribigbe@gmail.com

Authors' contribution:

A Study design · B Data collection · C Statistical analysis · D Data interpretation · E Manuscript preparation · F Literature search · G Funds collection 
differences between hemiplegic and non-hemiplegic limbs of stroke patients are based on the structural changes in the joints and soft tissues of the upper limb. Only a few researchers have studied sonographic arthritic changes in the knee joints of stroke patients [3,7-10]. Also, to the best of the authors' knowledge, none of the studies published in English literature has described structural sonographic determinant of knee joint pain in stroke patients.

Although magnetic resonance imaging (MRI) is accurate and reproducible for evaluating bone, articular cartilage, and soft tissues [11-13], it is expensive, time consuming, and not widely available for routine use in resource-poor settings. Arthroscopy provides a direct view of the cartilage and synovium and is considered as a gold standard for assessing cartilage lesions [14]. Its disadvantage is that it is an invasive procedure. Musculoskeletal ultrasound (USS) on the other hand has many advantages, including its noninvasive nature, low cost, portability, dynamic real-time assessment, and easy side-to-side comparison [15-17].

Thus, this study is aimed at evaluating the soft tissue arthritic changes on USS that are associated with pain in hemiplegic knees of stroke patients in our environment.

\section{Material and methods}

This case-controlled, cross-sectional study was carried out on 48 stroke and 26 apparently healthy subjects, who gave written, informed consent to participate in the study. Approval to carry out this research was obtained from the Ethic and Research Committee of the Obafemi Awolowo University Teaching Hospitals Complex (Protocol Number: ERC/2016/06/13; Approval Date: June 10, 2016). The procedures followed were in accordance with the Helsinki Declaration of 1975, as revised in 2000.

\section{Patient selection}

Forty-eight stroke patients (confirmed on brain computed tomography [CT] and/or brain MRI) presenting in the neurology out-patient clinic of the OAUTHC (Ile-Ife hospital unit) were recruited consecutively. Patients were included if they had a first episode of ischaemic or haemorrhagic unilateral stroke with hemiparesis and the ability to walk before stroke. Hemiplegic stroke patients with amputated lower limb(s) and patients who had inflammatory arthritis or other rheumatic diseases, a history of knee trauma (with involvement of the cruciate ligaments or menisci), or had undergone previous knee surgery were excluded. Twenty-six (26) age-, sex-, and body mass index (BMI)-matched control subjects with no evidence of stroke, knee disease, knee trauma, or knee surgery were recruited from patients' relatives and volunteers. Subjects who had knee arthrocentesis or an intra-articular steroid injection in the last three months were also excluded from the control group.

\section{Demographics and outcome measures}

The demographic features of the patients, e.g. age, sex, occupation, BMI, presence, and duration and severity of knee joint pain, were recorded.

Then, the tone in the knee flexor and the power in the knee extensor muscles were graded using Modified Ashworth (MA) and Medical Research Council (MRC) scales, respectively, while the patient's walking ability was graded using Functional Ambulation Category (FAC) described by Holden et al. [18]. To ensure optimal data analysis in this study, the tone in the knee joint was graded as 'normal' (MA scale $=0$ ) or 'increased tone' (MA scale $\geq 1$ ), power was grade as 'full' (MRC scale $=5$ ) or 'reduced' (MRC scale $\leq 4$ ), while FAC was graded as 'independent ambulator' or 'dependent and non-functional ambulator' $(\mathrm{FAC}<4)$.

\section{Ultrasound technique}

Using a high-frequency linear USS probe (7.5-12 MHz), both knees of the 48 hemiplegic stroke patients and 26 age-, sex- and BMI-matched healthy control subjects were examined. Sonographic examination was performed according to standardised scanning method $[15,16]$.

To examine the suprapatellar pouch and patellar tendon, the patient was placed in a supine position with knee flexion of $30^{\circ}$. The transducer was swept along the suprapatellar pouch, from medial to lateral or lateral to medial, to give a longitudinal image. The maximal synovial thickness and the maximum antero-posterior depth of the effusion was measured using the longitudinal axis (Figure 1A). With full flexion of the knee, the articular cartilage of the femoral trochlea was examined. The probe was placed in the transverse plane just above the superior margin of the patella. The thickness of the femoral condylar cartilage was then measured in the medial condyles and lateral condyles. The measurement was taken from the thin hyperechoic line at the soft tissue-cartilage interface to the hyperechoic line at the cartilage-bone interface (Figure 2A), while irregularities of these interfaces were noted if present. Three measurements were taken in each condyle (i.e. in the medial and lateral aspects of the condyle), and the mean value was calculated and recorded as the cartilage thickness.

For evaluation of the medial knee, the subject, while supine, was asked to rotate the leg externally with the knee flexed at $20-30^{\circ}$. The transducer is then placed in the coronal plane to evaluate the length of the medial collateral ligament and the body of the medial meniscus. Afterwards, the probe was gently advanced distally to demonstrate the tendons of the pes anserinus (Figure 3A). Longitudinal and transverse scans of the lateral collateral ligament and the body of the lateral meniscus were performed with the subject supine, the leg internally rotated, and with knee flexion of $20-30^{\circ}$. The probe was placed in the coronal-oblique plane. 

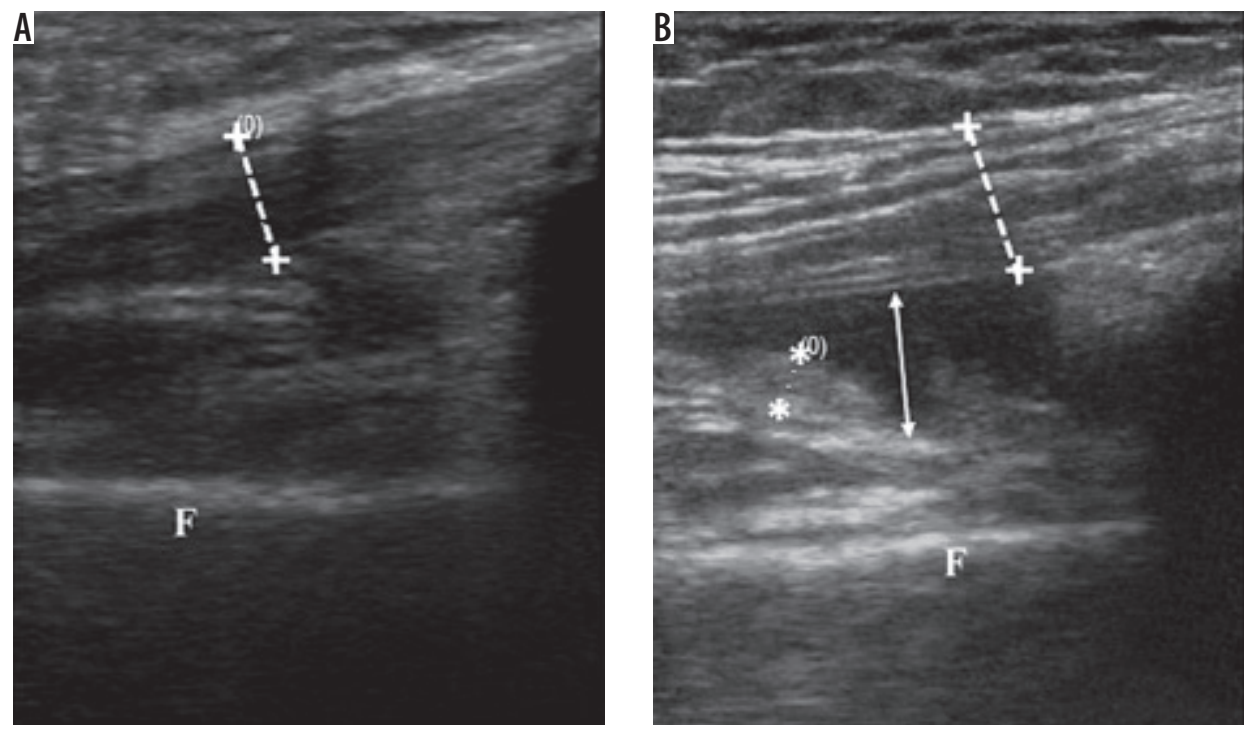

Figure 1. Sagital sonogram of anterior knee. A) Normal subject. B) Knee effusion (white arrow) and synovial hypertrophy $\left({ }^{* *}\right)$. F - femur, ++ - quadriceps tendon
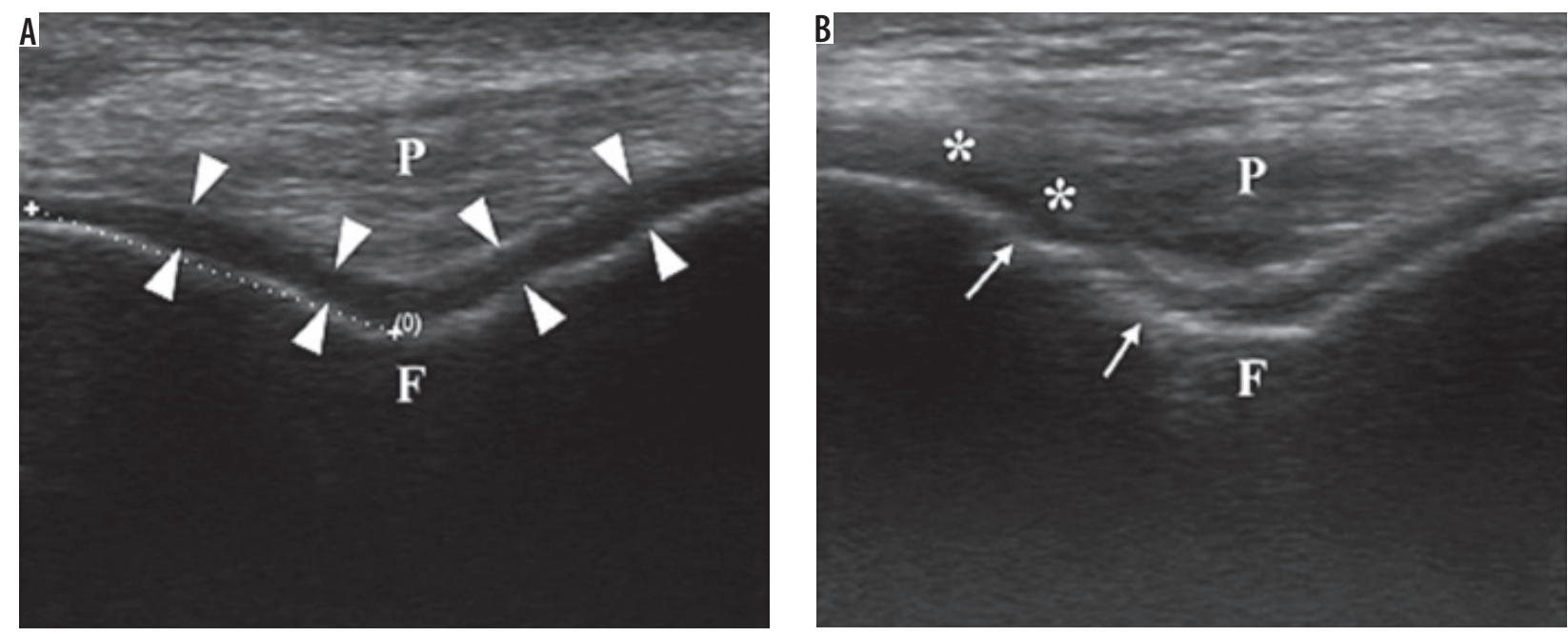

Figure 2. Transverse sonogram of anterior knee. A) Femoral hyaline cartilage (arrow heads) in a normal subject. B) Thinning of articular cartilage of the knee with blurring of cartilage-soft tissue interface ${ }^{* *}$ ) and irregular cartilage-bone interface (arrows). $\mathrm{F}$ - femur, $\mathrm{P}$ - patellar tendon in transverse section
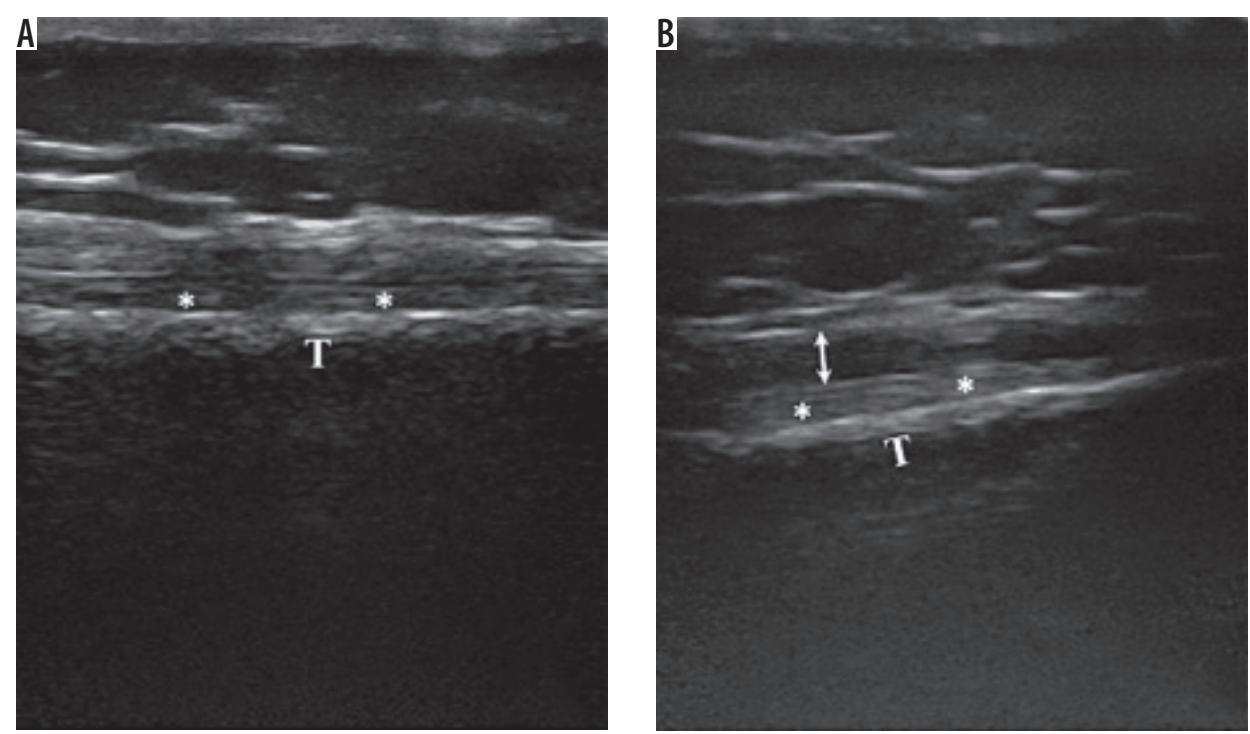

Figure 3. A) Pes anserinus tendon in a normal subject showing normal fibrillary pattern. B) Pes anserinus tendinosis showing mild thickening of the pes anserinus tendon with decreased fibrillary echogenicity. $T$ - tibia, ${ }^{* *}$ - medial collateral ligament 
To evaluate the posterior knee, the subject was asked to lie prone on the couch with the knee in full extension. The examination was commenced by scanning in the transverse plane over the mid-calf. The transducer was then moved proximally over the medial aspect of the posterior knee to evaluate the gastrocnemius-semimembranosus bursa. Both longitudinal and transverse scans were performed.

The same researcher (AAS) carried out the examinations to eliminate inter-observer variability. Sonographic measurements were taken three times, and the mean values were recorded for each subject.

\section{Sonographic criteria}

All disease processes were based on those widely described in the literature. Synovial hypertrophy was defined as an abnormal hypoechoic or anechoic intra-articular tissue that was non-displaceable and poorly compressible and could exhibit Doppler signal (Figure 1B). Synovial hypertrophy was said to be present when maximum synovial thickness in the longitudinal plane measured $\geq 4 \mathrm{~mm}$. Effusion was defined as an abnormal hypoechoic or anechoic intra-articular tissue that was compressible and displaceable and did not exhibit Doppler signal (Figure 1B). Effusion was considered present if the maximal depth in the longitudinal plane was $\geq 4 \mathrm{~mm}$. Meniscal protrusion was present when the distance between the peripheral border of the meniscus and the outline of the tibial plateau measured $>3 \mathrm{~mm}$. Pes anserinus tendinosis (PAT) was characterised by thickening and loss of the normal fibrillary echotexture [19,20]. Baker's cyst was identified when the gastrocnemius-semimembranosus bursa was filled with hypoechoic material and had a transverse diameter of $>4 \mathrm{~mm}$. Articular cartilage of the femoral trochlear was considered reduced when its mean thickness was $<2 \mathrm{~mm}$ [21] (Figure 3B).

Statistical Package for Social Sciences (SPSS INC USA) for windows Version 20.0 was used to analyse the data collected. Normally distributed numerical variables such as age and BMI were presented as mean \pm standard deviation for each of the study groups, while the independent sample $t$-test was used to compare the distribution. Non-normal distribution, as for duration of stroke and duration of knee pain, were presented as medians (interquartile range) while Friedman's two-way analysis of variance by ranks was used to compare the distributions. Categorical variables like the gender and the presence of arthritic changes among the study groups were presented and compared on contingency tables using $\chi^{2}$, Fisher's exact test, or McNemar test, as appropriate. The level of significance was set at $p<0.05$.

\section{Results}

The 48 patients had a mean age of $60.9 \pm 13.0$ years (range, 28-86 years) and a male-to-female ratio of 1.2 . The time from stroke onset ranged from 1 to 36 months (median $=12.5$ months). The 26 control subjects had a mean age of 61.4 \pm 12.1 years (range, 36-86 years) and a male-tofemale ratio of 1 . There were no significant differences among stroke patients and control subjects in terms of age $(p=0.927)$, gender distribution $(p=0.677)$, and body mass index $(p=0.142)$ (Table 1$)$.

Pain was reported more often in the hemiplegic than in the non-hemiplegic knees, with prevalences of 33.3\% and $12.5 \%$, respectively $(p=0.021$ ). Also, $5.8 \%$ (three subjects) reported pain in both knees while $36.5 \%$ (19 subjects) had no knee pain. Subjects with knee pain reported mild or moderate severity, while none of the subjects reported severe or disabling knee pain.

Median (Q1-Q3) duration of stroke was 11.5 (4.3-24.0) months, while median (Q1-Q3) durations of knee pain were 6.5 (1.9-22.3) months and 5.5 (4.0-15.0) months in the hemiplegic and non-hemiplegic knees, respectively. The duration of knee pain had no significant association with duration of stroke ( $p=0.368)$.

Positive changes were found in 44 (91.7\%) hemiplegic knees, 41 (85.4\%) non-hemiplegic knees, and 36 (69.2\%) control knees. The prevalence of sonographic arthritic changes was significantly higher in the hemiplegic than in the control knees $(p=0.005)$, and this difference is accounted for by supra patellar effusion $(p=0.036)$ and reduced lateral femoral cartilage $(p=0.004)$. The frequency of these soft-tissue arthritic changes are, however, similar between hemiplegic and non-hemiplegic knee arthritic changes ( $p=0.375)$ of stroke subjects.

In bivariate logistic regression analysis, clinical parameters such as age, gender, obesity, history of diabetes, and duration of hemiplegia showed no statistically significant relationship with hemiplegic knee pain (HKP). Also, there was no statistically significant relationship between HKP and functional outcome parameters such as tone in the knee flexors, power in the knee extensors, and functional ambulatory category. Among the sonographic arthritic changes evaluated, only pes anserinus tendinosis (PAT) showed an independent relationship with HKP in a multivariate binary logistic regression, with an odds ratio of 10 (CI: 1.7-61).

\section{Discussion}

This study has shown that hemiplegic knee pain (HKP) is common in stroke survivors, with a prevalence of $33.3 \%$. This is higher than the prevalence reported by Park et al. [10] (30\%) in a Korean population, Caglar et al. [4] (25.4\%) in a Turkish population, and Kong et al. [5] (6.5\%) in a Singaporean population. Similarly, non-HKP was reported in $12.5 \%$ of stroke subjects in the index study, which is also higher than that reported by Kong et al. [5] (2\%). While the average duration of stroke in the index study is similar to the mean duration in the Caglar et al. study [4] (11.1 months). Kong et al. [5] reported a higher mean duration (19.8 months), while Park et al. [10] studied more 
Table 1. Subject characteristics

\begin{tabular}{|c|c|c|c|c|}
\hline Variables & $\begin{array}{l}\text { Control } \\
n=26\end{array}$ & $\begin{array}{l}\text { Stroke } \\
n=48\end{array}$ & Statistics & $p$-value \\
\hline Age in years, mean $\pm D S$ & $61.4 \pm 12.0$ & $60.9 \pm 13.0$ & $-0.228^{*}$ & $0.918^{*}$ \\
\hline \multicolumn{5}{|l|}{ Age in group, $n(\%)$} \\
\hline$<40$ & $1(3.8)$ & $2(4.2)$ & \multirow[t]{5}{*}{$0.886^{\#}$} & \multirow[t]{5}{*}{0.927} \\
\hline $40-49$ & $3(11.5)$ & $5(10.4)$ & & \\
\hline $50-59$ & $8(30.8)$ & $13(27.1)$ & & \\
\hline $60-69$ & $6(23.1)$ & $15(31.2)$ & & \\
\hline$\geq 70$ & $8(30.8)$ & $13(27.1)$ & & \\
\hline \multicolumn{5}{|l|}{ Gender, $n(\%)$} \\
\hline Male & $13(50.0)$ & $26(54.2)$ & \multirow[t]{2}{*}{$0.174^{\#}$} & \multirow[t]{2}{*}{$0.677^{\#}$} \\
\hline Female & $13(50.0)$ & $22(45.8)$ & & \\
\hline BMI, mean \pm DS & $24.7 \pm 3.5$ & $24.4 \pm 4.0$ & $-0.228^{*}$ & $0.918^{*}$ \\
\hline \multicolumn{5}{|l|}{ BMI group, $n(\%)$} \\
\hline Underweight & $1(3.8)$ & $2(4.3)$ & \multirow[t]{4}{*}{$5.452^{\#}$} & \multirow[t]{4}{*}{0.142} \\
\hline Normal weight & $10(38.5)$ & $29(61.7)$ & & \\
\hline Overweight & $13(50.0)$ & $11(23.4)$ & & \\
\hline Obese & $2(7.7)$ & $5(10.6)$ & & \\
\hline Duration of stroke in months, median (IQR) & - & $11.5(4.3-24.0)$ & - & - \\
\hline \multicolumn{5}{|l|}{ Modified Ashworth scale, $n(\%)$} \\
\hline 0 (normal tone) & $29(60.4)$ & - & - & - \\
\hline$\geq 1$ (increased tone) & $19(39.6)$ & - & - & - \\
\hline \multicolumn{5}{|l|}{ Medical Research Council scale, $n(\%)$} \\
\hline 5 (full power) & $31(64.6)$ & - & - & - \\
\hline$\leq 4$ (reduced power) & $17(35.4)$ & - & - & - \\
\hline \multicolumn{5}{|l|}{ Functional ambulatory category, $n(\%)$} \\
\hline$\geq 4$ (independent ambulators) & $24(50.0)$ & - & - & - \\
\hline$\leq 3$ (dependent and non-functional ambulators) & $24(50.0)$ & - & - & - \\
\hline
\end{tabular}

*Independent sample $t$-test; ${ }^{*} \chi^{2}$ test statistic, IQR - interquartile range

recent stroke cases with a mean duration of six months. The variations in HKP prevalence among these studies may be due to differences in study design, disease severity, and ethno-racial disparity.

Positive findings were seen in about 91.7\% (hemiplegic knee) and $85.4 \%$ (non-hemiplegic knee) of stroke survivors studied. This is similar to the study by Yang et al. [3], who reported positive sonographic findings in $91.5 \%$ (hemiplegic knees) and $86.4 \%$ (non-hemiplegic knees) in a Chinese population. Suprapatellar effusion, reduced femoral cartilage thickness, irregular cartilage-bone margin, and blurred cartilage-soft tissue margin are the commonest soft tissues arthritic changes reported in the index study. Yang et al. [3] similarly identified suprapatellar effusion, patellar tendinitis, and blurred cartilage-soft tissue margin as the commonest arthritic changes. Among these described arthritic changes, only suprapatellar effusion and reduced

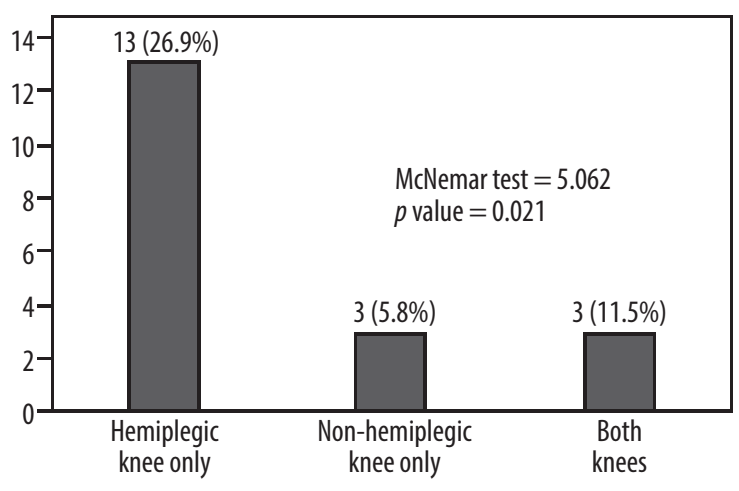

Figure 4. Prevalence of knee pain in the hemiplegic and non-hemiplegic limbs of stroke patients

lateral femoral cartilage thickness showed significant prevalence in the hemiplegic knees of stroke subjects compared to the controls. Tunç et al. [8] and Park et al. [10] also re- 
Table 2. Comparing soft tissue arthritic changes among stroke patients and healthy controls

\begin{tabular}{|l|c|c|c|c|c|c|}
\hline & Vemiplegic knee (H) & Non-hemiplegic knee $(\mathrm{nH})$ & Control (C) & \multicolumn{3}{|c|}{$p$-values } \\
\cline { 5 - 7 } & $n(\%)=52(100)$ & $n(\%)=52(100)$ & $n(\%)=52(100)$ & H vs. nH* & H vs. C\# & nH vs. C\# \\
\hline Presence of soft-tissue arthritic changes & $44(91.7)$ & $41(85.4)$ & $36(69.2)$ & 0.375 & 0.005 & 0.055 \\
\hline Suprapatellar effusion & $16(33.3)$ & $10(20.8)$ & $8(15.4)$ & 0.180 & 0.036 & 0.479 \\
\hline Suprapatellar synovitis & $3(6.2)$ & $5(10.4)$ & $1(1.9)$ & 0.687 & 0.348 & 0.102 \\
\hline Medial meniscal protrusion & $2(4.2)$ & $3(6.2)$ & $1(1.9)$ & 1.000 & 0.606 & 0.348 \\
\hline Lateral meniscal protrusion & $4(8.3)$ & $5(10.4)$ & $4(7.7)$ & 1.000 & 1.000 & 0.734 \\
\hline Reduced medial femoral cartilage thickness & $21(43.8)$ & $20(41.7)$ & $21(40.4)$ & 1.000 & 0.733 & 0.896 \\
\hline Reduced lateral femoral cartilage thickness & $22(45.8)$ & $17(35.4)$ & $10(19.2)$ & 0.267 & 0.004 & 0.069 \\
\hline Blurred cartilage-soft tissue margin & $12(25.0)$ & $8(16.7)$ & $8(15.4)$ & 0.289 & 0.230 & 0.861 \\
\hline Irregular cartilage-bone margin & $14(29.2)$ & $13(27.1)$ & $13(25.0)$ & 1.000 & 0.512 & 0.824 \\
\hline Pes anserinus tendinosis & $9(18.8)$ & $11(22.9)$ & $4(7.7)$ & 0.687 & 0.100 & 0.033 \\
\hline Femoral osteophytes & $31(64.6)$ & $31(64.6)$ & $25(48.1)$ & 1.000 & 0.097 & 0.097 \\
\hline Tibial osteophytes & $29(60.4)$ & $32(66.7)$ & $24(46.2)$ & 0.375 & 0.153 & 0.046 \\
\hline Baker's cyst & $1(2.1)$ & $0(0.0)$ & $0(0.0)$ & - & 0.480 & - \\
\hline
\end{tabular}

${ }^{*}$ McNemar test statistic; ${ }^{*} \chi^{2} /$ Fisher's exact test; the $p$ values in bold are significant

ported reduced mean lateral femoral cartilage thickness (LFC) in the hemiplegic knees compared to the LFC in the non-hemiplegic knees of stroke subjects. Because femoral cartilage is thicker in contact regions [22,23], characteristic excessive medial knee loading, which has been described in both knees of hemiplegic stroke patients, compared to healthy controls, may be responsible for reduced lateral femoral cartilage thickness in the hemiplegic knees [24].

In our study, the comparison of arthritic changes between the hemiplegic and non-hemiplegic knees of stroke patients showed no statistically significant difference (Table 2). Conversely, Yang et al. [3] reported a statistically significant increased prevalence of suprapatellar effusion and patellar tendinitis in hemiplegic compared to non-hemiplegic knees, while Tunç et al. [8] reported statistically significant reduction in the lateral femoral cartilage thickness in the hemiplegic knees compared to the non-hemiplegic knees of stroke patients. Park et al. [10] also reported PAT, reduced intercondylar cartilage thickness, and reduced lateral condylar cartilage loss ratio in the hemiplegic knees. These variations may be due to gender disparity (the male gender proportion is lower in the index study compared to Yang et al. [3], Tunç et al. [8], and Park et al. [10]) and/or differences in intensity of rehabilitation in the different study cohorts.

Clinical variables (like age, gender, stroke duration, and presence of diabetes mellitus) and outcome parameters (like tone, power, and FAC) showed no association with HKP (Table 3). This also agrees with the study by Caglar et al. [4], who reported no statistically significant association between hemiplegic shoulder/knee pain and Brunnstrom motor evaluation and functional independence measurement scores. This may suggest that other fac- tors during rehabilitation, such as inappropriate physical therapy, may be responsible for HKP.

Although both blurred cartilage-soft tissue margin and PAT showed positive association with HKP, only the latter proved to be an independent predictor in our study cohort, with an odds ratio of 10 (Table 3 ). This implies that stroke patients with PAT have 10-fold increased risk of HKP compared to stroke patients without PAT. The prevalence of PAT in the index study (18.8\% and $22.9 \%$ in the hemiplegic and non-hemiplegic knees, respectively) is similar to that reported by Yang et al. [3] (27\% and 20\% in the hemiplegic and non-hemiplegic knees, respectively). However, to the best of our knowledge, this study is the first to describe the statistically significant relationship between HKP and PAT.

A possible explanation for this observation is the excessive medial knee loading in hemiplegic stroke patients compared to healthy controls (see above) either during physical rehabilitation or ambulation. In addition, excessive loading has been reported in both the paretic and non-paretic legs of stroke patients [24]. These biomechanical changes can cause stress/injury to the soft tissues, especially in the medial knee, including the pes anserinus tendon.

A prospective, double-blind, randomised, controlled trial by Cho et al. [9] evaluated the effects of weekly extracorporeal shockwave therapy (ESWT) on some sonographic arthritic changes of 18 chronic stroke patients over a three-week period. Although they reported a statistically significant increased Doppler activity (increased blood flow) in the medial knee joint in the experimental group, there was no statistically significant change in femoral articular cartilage thickness and joint effusion height. 
Table 3. The relationship between some clinical parameters, sonographic soft tissue arthritic changes, and hemiplegic knee pain

\begin{tabular}{|c|c|c|c|c|c|c|}
\hline \multirow[t]{2}{*}{ Variables } & \multicolumn{2}{|c|}{ Hemiplegic knee pain } & \multicolumn{2}{|c|}{ Univariate } & \multicolumn{2}{|c|}{ Multivariate } \\
\hline & $\begin{array}{c}\text { Absent } \\
n=32(100 \%)\end{array}$ & $\begin{array}{c}\text { Present } \\
n=20(100 \%)\end{array}$ & $p$ value & $\begin{array}{c}\text { OR } \\
(95 \% \mathrm{Cl})\end{array}$ & $p$ value & $\begin{array}{c}\text { OR } \\
(95 \% \mathrm{CI})\end{array}$ \\
\hline Age ( $\geq 60$ years) & $17(53.1)$ & $11(68.8)$ & 0.301 & $1.9(0.5-6.9)$ & - & - \\
\hline Gender (female) & $16(50.0)$ & $6(37.5)$ & 0.482 & $0.6(0.2-2.0)$ & - & - \\
\hline Obesity & $4(12.5)$ & $1(6.2)$ & 0.504 & $0.5(0.0-4.6)$ & - & - \\
\hline Diabetes & $8(25.0)$ & $4(25.0)$ & 1.000 & $0.8(0.2-2.9)$ & - & - \\
\hline Stroke duration ( $\geq 12$ months) & $18(56.2)$ & $6(37.5)$ & 0.221 & $0.5(0.1-1.6)$ & - & - \\
\hline Increased tone & $14(43.8)$ & $5(31.2)$ & 0.404 & $0.6(0.2-2.1)$ & - & - \\
\hline Reduced power & $12(37.5)$ & $6(30.0)$ & 0.670 & $0.8(0.2-2.7)$ & - & - \\
\hline FAC (dependent/non-ambulators) & $14(43.8)$ & $10(62.5)$ & 0.221 & $2.1(0.6-7.3)$ & - & - \\
\hline Suprapatellar effusion & $11(34.4)$ & $5(31.2)$ & 0.829 & $0.9(0.2-3.1)$ & - & - \\
\hline Suprapatellar synovitis & $2(6.2)$ & $1(6.2)$ & 1.000 & $1.0(0.1-12)$ & - & - \\
\hline Medial meniscal protrusion & $2(6.2)$ & $0(0.0)$ & 0.546 & - & - & - \\
\hline Lateral meniscal protrusion & $4(12.5)$ & $0(0.0)$ & 0.286 & - & - & - \\
\hline Reduced medial femoral cartilage & $15(46.9)$ & $6(37.5)$ & 0.537 & $0.7(0.2-2.3)$ & - & - \\
\hline Reduced lateral femoral cartilage & $14(43.8)$ & $8(50.0)$ & 0.682 & $1.3(0.4-4.3)$ & - & - \\
\hline Blurred cartilage-soft tissue margin & $5(15.6)$ & $7(43.8)$ & 0.034 & $3.6(1.1-17)$ & 0.107 & $3.4(0.8-16)$ \\
\hline Irregular cartilage-bone margin & $10(31.2)$ & $4(25.0)$ & 0.746 & $0.7(0.2-2.8)$ & - & - \\
\hline Pes anserinus tendinosis & $2(6.2)$ & $7(43.8)$ & 0.004 & $12(2.1-66)$ & 0.028 & $10(1.7-61)$ \\
\hline Baker's cyst & $0(0.0)$ & $0(0.0)$ & - & - & - & - \\
\hline Femoral osteophytes & $20(62.5)$ & $11(68.8)$ & 0.670 & $1.3(0.4-4.7)$ & - & - \\
\hline Tibial osteophytes & $19(59.4)$ & $10(62.5)$ & 0.835 & $1.1(0.3-3.9)$ & - & - \\
\hline
\end{tabular}

$\mathrm{OR}$ - odds ratio, $\mathrm{Cl}$ - confidence interval; the $p$ values in bold are significant

They also observed that ESWT reduced knee pain and improved function in stroke patients.

A comparison of MRI and high-frequency ultrasound in evaluating 106 patients with post-stroke shoulder joint pain by Zaiton et al. [25] showed that ultrasound provided high diagnostic accuracy and was inexpensive. Similar high diagnostic accuracy was reported in the evaluation of knee joints of patients with osteoarthritis and rheumatoid arthritis [26]. However, most published MRI-based studies evaluating lower limb functions in stroke patients focused more on stroke-related sarcopaenia in the thigh and leg muscles $[27,28]$.

The limitations of this study include selection bias because only subjects presenting in the hospital were recruited. Also, the ultrasound operator was not completely blinded to all of the subjects' clinical details, and the frequency and type of rehabilitation were not assessed.

\section{Conclusions}

There is a paucity of studies on the role of diagnostic ultrasound in evaluating knee joints of stroke survivors.
This study demonstrated a significant increase in sonographic arthritic changes in the hemiplegic and non-hemiplegic knees of stroke subjects compared to healthy control knees. We also found that stroke patients with PAT have 10-fold greater risk of HKP compared to those without PAT. Routine knee joint assessment with ultrasound is recommended to identify treatable arthritic changes and to monitor response to treatment in stroke survivors, while patients' rehabilitation should incorporate the findings as well as include measures to reduce pain and arthritis so as to improve their quality of life. A multidisciplinary approach to rehabilitation is also recommended. Further prospective studies could include observation of the effects of the treatment of HKP on quality of life as well as other outcome measures such as the Modified Ranking Scale.

\section{Conflict of interest}

The authors declare no conflict of interest. 


\section{References}

1. Chen CL, Chen HC, Tang SFT, Wu CY, Cheng PT, Hong WH. Gait performance with compensatory adaptations in stroke patients with different degrees of motor recovery. Am J Phys Med Rehabil 2003; 82: 925-935.

2. Richards JD, Pramanik A, Sykesand L, Pomeroy VM. A comparison of knee kinematic characteristics of stroke patients and age-matched healthy volunteers. Clin Rehabil 2003;17: 565-571.

3. Yang CP, Lee CL, Chen TW, Lee S, Weng MC, Huang MH. Ultrasonographic findings in hemiplegic knees of stroke patients. Kaohsiung J Med Sci 2005; 21: 70-77.

4. Caglar NS, Akin T, Aytekin E, et al. Pain syndromes in hemiplegic patients and their effects on rehabilitation results. J Phys Ther Sci 2016; 28: 731-737.

5. Kong KH, Woon VC, Yang SY. Prevalence of chronic pain and its impact on health-related quality of life in stroke survivors. Arch Phys Med Rehabil 2004; 85: 35-40.

6. Kumar P. Hemiplegic shoulder pain in people with stroke: present and the future. Pain Manag 2019; 9: 107-110.

7. Lee CL, Chen TW, Weng MC, Wang YL, Cheng HS, Huang MH. Ultrasonographic findings in hemiplegic shoulders of stroke patients. Kaohsiung J Med Sci 2002; 18: 70-76.

8. Tunç H, Öken Ö, Kara M, et al. Ultrasonographic measurement of the femoral cartilage thickness in hemiparetic patients after stroke. Int J Rehabil Res 2012; 35: 203-207.

9. Cho SJ, Yang JR, Yang HS, Yang HE. Effects of extracorporeal shockwave therapy in chronic stroke patients with knee osteoarthritis: a pilot study. Ann Rehabil Med 2016; 40: 862-870.

10. Park SA, Yang CY, Kim JH, Lee KK, Shin BC, Lee I. Ultrasonographic findings of both knee in hemiplegic ambulators with recent stroke. Korean J Oriental Physiol Pathol 2012; 26: 539-545.

11. Cook JL, Cook CR, Stannard JP, et al. MRI versus ultrasonography to assess meniscal abnormalities in acute knees. J Knee Surg 2014; 27: 319-324.

12. Hemke R, Kuijpers TW, van den Berg JM, et al. The diagnostic accuracy of unenhanced MRI in the assessment of joint abnormalities in juvenile idiopathic arthritis. Eur Radiol 2013; 23: 1998-2004.

13. Sharifah M, Lee C, Suraya A, Johan A, Syed A, Tan S. Accuracy of MRI in the diagnosis of meniscal tears in patients with chronic ACL tears. Knee Surg Sports Traumatol Arthrosc 2015; 23: 826-830.

14. Karim Z, Wakefield R, Quinn M, et al. Validation and reproducibility of ultrasonography in the detection of synovitis in the knee: a comparison with arthroscopy and clinical examination. Arthritis Rheum 2004; 50: 387-394.

15. Alves TI, Girish G, Kalume Brigido M, Jacobson JA. US of the knee: scanning techniques, pitfalls, and pathologic conditions. Radiographics 2016; 36: 1759-1775
16. De Maeseneer M, Marcelis S, Boulet C, et al. Ultrasound of the knee with emphasis on the detailed anatomy of anterior, medial, and lateral structures. Skeletal Radiol 2014; 43: 1025-1039.

17. Mureşan S, Mureşan M, Voidăzan S, Neagoe R. The accuracy of musculoskeletal ultrasound examination for the exploration of meniscus injuries in athletes. J Sports Med Phys Fitness 2017; 57: 589594.

18. Holden MK, Gill KM, Magliozzi MR, Nathan J, Piehl-Baker L. Clinical gait assessment in the neurologically impaired: reliability and meaningfulness. Phys Therapy 1984; 64: 35-40.

19. Uysal F, Akbal A, Gökmen F, Adam G, Reşorlu M. Prevalence of pes anserine bursitis in symptomatic osteoarthritis patients: an ultrasonographic prospective study. Clin Rheumatol 2015; 34: 529-533.

20. Toktas H, Dundar U, Adar S, Solak O, Ulasli AM. Ultrasonographic assessment of pes anserinus tendon and pes anserinus tendinitis bursitis syndrome in patients with knee osteoarthritis. Modern Rheumatol 2015; 25: 128-133.

21. Çarlı AB, Akarsu S, Tekin L, Sağlam M, Kıralp MZ, Özçakar L. Ultrasonographic assessment of the femoral cartilage in osteoarthritis patients with and without osteoporosis. Aging Clin Exp Res 2014; 26: 411-415.

22. Li G, Park SE, DeFrate LE, et al. The cartilage thickness distribution in the tibiofemoral joint and its correlation with cartilage-to-cartilage contact. Clin Biomech (Bristol, Avon) 2005; 20: 736-744.

23. Li G, Lopez O, Rubash H. Variability of a three-dimensional finite element model constructed using magnetic resonance images of a knee for joint contact stress analysis. J Biomech Eng 2001; 123 : 341-346.

24. Marrocco S, Crosby L, Jones IC, Moyer RF, Birmingham TB, Patterson KK. Knee loading patterns of the non-paretic and paretic legs during post-stroke gait. Gait Posture 2016; 49: 297-302.

25. Zaiton F, Tantawy HI, Elsharkawy KAM, Sanad G. Painful poststroke shoulder: comparison of magnetic resonance imaging and high frequency ultrasonography. Egyptian J Radiol Nucl Med 2011; 42: 47-55.

26. Sadeghian H, Shekarchi B, Niya MZ, Bagheri H. Ultrasound findings in knee osteoarthritis and rheumatoid arthritis compared to MRI. Bali Med J 2018; 7: 468-471.

27. Silva-Couto MdA, Lanatovitz Prado-Medeiros Ch, Oliveira AB, et al. Muscle atrophy, voluntary activation disturbances, and low serum concentrations of IGF-1 and IGFBP-3 are associated with weakness in people with chronic stroke. Phys Ther 2014; 94: 957967.

28. Hunnicutti JL, Gregory CM. Skeletal muscle changes following stroke: a systematic review and comparison to healthy individuals. Top Stroke Rehabil 2017; 24: 463-471. 